\title{
Preface to Volume 2021, Issue 1
}

\author{
Itai Dinur $^{1}$ and Gaëtan Leurent ${ }^{2}$ \\ ${ }^{1}$ Ben-Gurion University, Beer Sheva, Israel \\ ${ }^{2}$ Inria, Paris, France
}

IACR Transactions on Symmetric Cryptology (ToSC) is a forum for original results in all areas of symmetric cryptography, including the design and analysis of block ciphers, stream ciphers, encryption schemes, hash functions, message authentication codes, (cryptographic) permutations, authenticated encryption schemes, cryptanalysis and evaluation tools, and security issues and solutions regarding their implementation.

ToSC implements an open-access journal/conference hybrid model following some other communities in computer science. All articles undergo a journal-style reviewing process and accepted papers are published in gold open access (in our case the Creative Commons License CC-BY 4.0). The review procedures that we have followed strictly adhere to the traditions of the journal world.

The ToSC review process strives to maintain a high quality of published articles. Full papers are assigned to at least three members of the Editorial Board; for submissions by Editorial Board members this was increased to at least four. These members write detailed and careful reviews (usually without relying on subreviewers). Moreover, we have had a rebuttal phase, allowing authors to respond to the review comments before the final decisions. If necessary, the review process enables further interactions between the authors and the reviewers, mediated by the Co-Editors-in-Chief. The Editorial Board can also decide to ask for a minor or major revision of the paper when changes are deemed necessary to improve its quality. Furthermore, starting 2020, a "reject and resubmit" decision can be given in case the Editorial Board sees some potential in a paper, but there are significant issues to address before it can be properly evaluated.

Starting 2020, ToSC also accepts submissions of addendum and errata papers. Addendum papers aim at extending an existing ToSC paper in a novel, yet succinct way. Errata papers aim at correcting an error in an existing ToSC paper.

Overall, we are very pleased with the quality and quantity of submissions, the detailed review reports written by the reviewers and the substantial efforts by the authors to further improve the quality of their work. We think that the review process, and in particular the use of major revisions, leads to an increased quality of the papers that are published.

The papers selected by the Editorial Board for publication are presented at the conference Fast Software Encryption (FSE). This gives the authors the opportunity to advertise their results and engage in discussions on further work. The worldwide outbreak of COVID-19 has led to changes in the FSE schedule. FSE 2020 was held virtually in November 9-13, with paper from issues: 2019(2), 2019(3), 2019(4), 2020(1), 2020(2), 2020(3) and 2020(S1). FSE 2021 was canceled, while FSE 2022 will be held in Athens, Greece on March 20-25, with papers from the following issues of ToSC: 2020(4), 2021(1), 2021(2), 2021(3), 2021(4) and 2022(1). In addition to the scientific papers from the journal, FSE 2020 had two invited talks, by Kazuhiko Minematsu on security of OCB2 and Thomas Peyrin on tweakable block ciphers, and two Ask Me Anything sessions with Joan Daemen and Kaisa Nyberg.

Table 1 gives the submission statistics for issues 2020(2), 2020(3), 2020(4) and 2021(1). For example, for Volume 2020, Issue 3, we received 30 regular submissions, out of which 
Table 1: Submission statistics for issues 2020(2), 2020(3), 2020(4), 2021(1)

\begin{tabular}{|c|c|c|c|c|c|c|}
\hline $\begin{array}{c}\text { Volume } \\
\text { (Issue) }\end{array}$ & $\begin{array}{c}\text { Regular } \\
\text { Submissions }\end{array}$ & $\begin{array}{c}\text { Accepted } \\
\text { (Minor Revision) }\end{array}$ & $\begin{array}{c}\text { Major } \\
\text { Revision }\end{array}$ & $\begin{array}{c}\text { Decision } \\
\text { Deferred }\end{array}$ & $\begin{array}{c}\text { Reject and } \\
\text { Resubmit }\end{array}$ & Errata \\
\hline $2020(2)$ & 29 & $5(4)$ & 4 & 0 & 8 & 0 \\
$2020(3)$ & 30 & $10(4)$ & 5 & 0 & 3 & 1 \\
$2020(4)$ & 47 & $9(3)$ & 7 & 1 & 4 & 0 \\
$2021(1)$ & 37 & $13(5)$ & 5 & 0 & 8 & 0 \\
\hline
\end{tabular}

10 were accepted (including 4 minor revisions) and 5 papers received a major revision decision. The decision for none of the submissions was deferred to the next cycle. Out of the remaining rejected papers, 3 received a "reject and resubmit" decision. We further accepted one errata paper.

As it is tradition for FSE, the Editorial Board also selected best papers, based on the scientific quality and contribution. This year the Editorial Board has decided to give the award to two papers: "Catching the Fastest Boomerangs - Application to SKINNY" by Stéphanie Delaune, Patrick Derbez and Mathieu Vavrille, and "Cryptanalysis of LowMC instances using single plaintext/ciphertext pair" by Subhadeep Banik, Khashayar Barooti, F. Betül Durak and Serge Vaudenay.

We would like to thank the authors of all submissions for contributing high quality submissions. In particular, we would like to thank the Editorial Board members; we value their hard work and dedication to write constructive and detailed reviews and to engage in interesting discussions. Many Editorial Board members spent additional time as shepherds to help the authors improving their works.

We are deeply grateful of the work of Kevin McCurley and Kay McKelly who organized the virtual conference FSE 2020, and Christina Boura who had planned FSE 2020 in Athens, and will be the general chair of FSE 2022. We also would like to thank Anne Canteaut, Gregor Leander, Friedrich Wiemer, Phil Hebborn, and Linda Groß for their work and support. We hope that the papers in this volume of IACR Transactions on Symmetric Cryptology (ToSC) prove valuable and we are glad to see that ToSC is becoming the leading international venue publishing the top research on symmetric cryptology.

March 2021

Itai Dinur

Gaëtan Leurent 


\section{Editorial Board}

Elena Andreeva

Frederik Armknecht

Tomer Ashur

Subhadeep Banik

Zhenzhen Bao

Christof Beierle

Patrick Derbez

Christoph Dobraunig

Orr Dunkelman

Maria Eichlseder

Vincent Grosso

Jian Guo

Takanori Isobe

Tetsu Iwata

Jérémy Jean

Pierre Karpman

Nathan Keller

Stefan Kölbl

Virginie Lallemand

Gregor Leander

Jooyoung Lee

Stefan Lucks

Willi Meier

Brice Minaud

Kazuhiko Minematsu

Nicky Mouha

Kaisa Nyberg

Léo Perrin

Thomas Peyrin

Bart Preneel

Yann Rotella

Yannick Seurin

Siang Meng Sim

Hadi Soleimany

Ling Song

Siwei Sun
Technical University of Vienna, Vienna, Austria

University of Mannheim, Mannheim, Germany

KU Leuven, Leuven, Belgium

TU Eindhoven, Eindhoven, The Netherlands

Ecole Polytechnique Federale de Lausanne (EPFL), Lausanne, Switzerland

Nanyang Technological University (NTU), Singapore, Singapore

Ruhr University Bochum, Bochum, Germany

University of Rennes, Rennes, France

Centre national de la recherche scientifique (CNRS), Rennes, France

Institut de Recherche en Informatique et Systèmes Aléatoires (IRISA), Rennes, France

Lamarr Security Research, Graz, Austria

University of Haifa, Haifa, Israel

Graz University of Technology, Graz, Austria

University of Lyon, Saint-Étienne, France

Centre national de la recherche scientifique (CNRS), Saint-Étienne, France

Nanyang Technological University (NTU), Singapore, Singapore

University of Hyogo, Kobe, Japan

Nagoya University, Nagoya, Japan

Agence Nationale de la Sécurité des Systèmes d'Information (ANSSI), Paris, France

Université Grenoble Alpes, Grenoble, France

Bar-Ilan University, Ramat Gan, Israel

Google, Zurich, Switzerland

Centre National de la Recherche Scientifique (CNRS), Nancy, France

Ruhr University Bochum, Bochum, Germany

Korea Advanced Institute of Science and Technology (KAIST), Daejeon, South Korea

Bauhaus-Universität Weimar, Weimar, Germany

University of Applied Sciences and Arts Northwestern Switzerland (FHNW), Windisch, Switzerland

Inria, Paris, France

École Normale Supérieure (ENS), Paris, France

NEC, Kawasaki, Japan

National Institute of Standards and Technology (NIST), Gaithersburg, United States

Aalto University, Helsinki, Finland

Inria, Paris, France

Nanyang Technological University (NTU), Singapore, Singapore

KU Leuven, Leuven, Belgium

University of Versailles, Versailles, France

Agence Nationale de la Sécurité des Systèmes d'Information (ANSSI), Paris, France

DSO National Laboratories, Singapore, Singapore

Shahid Beheshti University, Teheran, Iran

Jinan University, Guangzhou, China

Chinese Academy of Sciences, Beijing, China 
Yosuke Todo Aleksei Udovenko Gilles Van Assche Damian Vizár

Qingju Wang
NTT Secure Platform Laboratories, Tokyo, Japan

CryptoExperts, Paris, France

STMicroelectronics, Diegem, Belgium

Centre suisse d'électronique et de microtechnique (CSEM), Neuchâtel, Switzerland

University of Luxembourg, Luxembourg, Luxembourg

\section{External reviewers}

Gustavo Banegas

Ritam Bhaumik

Federico Canale

André Chailloux

Akiko Inoue

Atul Luykx

Bart Mennink

María Naya-Plasencia

Patrick Neumann

Yu Sasaki

André Schrottenloher 